\title{
Psychiatric rehabilitation: a model to enhance return to work outcomes
}

Kristin Tugman

Unum, Portland ME 04101, USA. Ktugman@unum.com

Background: According to the world health organization by 2030 depression will rank first in economic burden in all high-income countries. As behavioural health disabilities continue to increase, disability managers will continue to be challenged to find ways to assist these workers in their effort to return to productivity.

Objectives: The objective of the discussion is to share a specific intervention strategy that disability managers can practically apply to effectively manage those individuals out of work on a behavioural/mental health claim. The process begins through using motivational interviewing to build a relationship so that the claims professional has the opportunity to recognize key intervention points throughout the absence from work. There are three components the disability manager should assess throughout the life of the event. The first is where the person is on the path to the disability mindset. This will help gain an understanding of current motivation for productivity. Disability is often experienced through specific consequence and those consequences can be both negative and positive. They evolve throughout the life of the leave. Recognition of the current consequence can help direct intervention. Finally specific cognitive barriers tend to emerge in the life of the disability such as faulty thinking, fear, or inactivity. If these barriers are not addressed the individual will not return to work.

Methods: A 20-person disability management team with associated clinical and vocational resources is using these techniques among an active block of over 1000 disability claimants. The work started in November of 2013 and there are currently very preliminary findings.

Findings: The results are positive with a reduction $\mathrm{n}$ closed duration days month over month. The technique will have been in place 1 year at the time of the conference and as a result more comprehensive data and outcomes will be shared. 